\title{
Colonization potential to reconstitute a microbe community in patients detected early after fecal microbe transplant for recurrent $C$. difficile
}

Ranjit Kumar ${ }^{1}$, Craig L. Maynard ${ }^{2}$, Peter Eipers ${ }^{3}$, Kelly T. Goldsmith ${ }^{4}$, Travis Ptacek ${ }^{1,5}$, J. Aaron Grubbs ${ }^{6}$, Paula Dixon ${ }^{6}$, Donna Howard ${ }^{2}$, David K. Crossman ${ }^{4}$, Michael R. Crowley ${ }^{4}$, William H. Benjamin Jr. ${ }^{2}$, Elliot J. Lefkowitz ${ }^{1,5}$,

Casey T. Weaver ${ }^{2}$, J. Martin Rodriguez ${ }^{6}$ and Casey D. Morrow ${ }^{3^{*}}$

\begin{abstract}
Background: Fecal microbiota transplants (FMT) are an effective treatment for patients with gut microbe dysbiosis suffering from recurrent $C$. difficile infections. To further understand how FMT reconstitutes the patient's gut commensal microbiota, we have analyzed the colonization potential of the donor, recipient and recipient post transplant fecal samples using transplantation in gnotobiotic mice.

Results: A total of nine samples from three human donors, recipient's pre and post FMT were transplanted into gnotobiotic mice. Microbiome analysis of three donor fecal samples revealed the presence of a high relative abundance of commensal microbes from the family Bacteriodaceae and Lachnospiraceae that were almost absent in the three recipient pre FMT fecal samples $(<0.01 \%)$. The microbe composition in gnotobiotic mice transplanted with the donor fecal samples was similar to the human samples. The recipient samples contained Enterobacteriaceae, Lactobacillaceae, Enterococcaceae in relative abundance of $43,11,8 \%$, respectively. However, gnotobiotic mice transplanted with the recipient fecal samples had an average relative abundance of unclassified Clostridiales of $55 \%$, approximately 7000 times the abundance in the recipient fecal samples prior to transplant. Microbiome analysis of fecal samples from the three patients early (2-4 weeks) after FMT revealed a microbe composition with the relative abundance of both Bacteriodaceae and Lachnospiraceae that was approximately $7 \%$ of that of the donor. In contrast, gnotobioitc mice transplanted with the fecal samples obtained from the three at early times post FMT revealed increases in the relative abundance of Bacteriodaceae and Lachnospiraceae microbe compositions to levels similar to the donor fecal samples. Furthermore, the unclassified Clostridiales in the recipient samples post FMT was reduced to an average of $10 \%$.

Conclusion: We have used transplantation into gnotobiotic mice to evaluate the colonization potential of microbiota in FMT patients early after transplant. The commensal microbes present at early times post FMT out competed non-commensal microbes (e.g. such as unclassified Clostridiales) for niche space. The selective advantage of these commensal microbes to occupy niches in the gastrointestinal tract helps to explain the success of FMT to reconstitute the gut microbe community of patients with recurrent $C$. difficile infections.
\end{abstract}

Keywords: Fecal microbiota transplant, Clostridium difficile, Niche, Gnotobiotic, Commensal

\footnotetext{
* Correspondence: caseym@uab.edu

${ }^{3}$ Department of Cell, Developmental and Integrative Biology, University of

Alabama at Birmingham, 1918 University Blvd. MCLM 680, Birmingham, AL

35294, USA

Full list of author information is available at the end of the article
} 


\section{Background}

Clostridium difficile infections are the major causative agent for infective antibiotic associated diarrhea [1, 2]. Infections are most commonly acquired in healthcare settings although community acquired infections are increasingly being reported [1]. In addition to recent use of antibiotics, other risk factors include old age, use of gastric acid suppressing drugs and underlying chronic disease including inflammatory bowel disease [1]. The numbers of infections have been rising during the last decade with estimated health care costs in the billions $[3,4]$.

The standard treatments for $C$. difficile infection consist of metronidazole, vancomycin, or fidaxomicin, which results in a rate of recurrence at about $20 \%$; after a third recurrence, the risk of further episodes is even higher [5-7]. Fecal microbiota transplantation (FMT) for treatment of recurrent $C$. difficile has had remarkable success rates for alleviation of the symptoms and restoration of health [8-12].

The reason why FMT is so effective in restoring a microbiome in the gastrointestinal tract of the patients is unknown. Presumably, an effective long-term stable reconstruction would require the commensal microbes in the FMT to access and occupy the niches in the gastrointestinal space following transplantation [13]. Although there have been numerous reports on the composition of the patients microbiota following FMT, there have been no studies to examine the potential of the donor microbes to colonize the recipients post transplant. To gain insights into this issue, we have examined this aspect of microbiome reconstruction following FMT by transplanting human fecal samples into gnotobiotic mice. Since these mice are devoid of microbes, previous studies have shown that the unoccupied (open) niches in the gastrointestinal tract readily accept fecal transplantation and recapitulate the major elements of the human microbiome in the gnotobiotic mice $[14,15]$. From the analysis of fecal samples from gnotobiotic mice with transplanted with donor, recipient and post FMT samples, we demonstrate that the microbiota of recipient early post FMT possess the capacity to reconstitute gnotobiotic mice with a microbiome community that is similar to the donor.

\section{Results}

Three patients that had undergone fecal transplants were chosen for this study. All of the three recipients were positive for $C$. difficile at least once and had undergone several rounds of antibiotic treatments without complete resolution of the repeated episodes of colitis. The characteristics of the recipients with respect to age, antibiotic treatment and comorbidities in addition to the $C$. difficile infection can be found in the Additional file 1: Data Set S1. Each recipient agreed to a fecal transplant with individual donors, usually a spouse or other family member. The transplants were accomplished by nasogastric administration. The details for preparation of the donor sample and administration can be found in Additional file 2: Text S1. For all three transplants, fecal samples were collected from donor, recipient and recipient post transplant (collected 24 weeks post fecal transplant). By convention, for a given specific transplant (e.g. transplant number 1) we refer to the donor (D), recipient (R) and Recipient post Transplant (RpT) with a prefix letter and the specific transplant number (e.g. D1, R1, RpT1). The Recipient post Transplant samples also carries a suffix which represent time post transplant i.e. RpT1w2 means that the sample is taken 2 week post fecal transplant. The human samples when transplanted in mouse carries a prefix "M" (for example when human sample RpT1w2 is transplanted in mouse it is named MRpT1w2). The list of all human and mouse samples used for microbiota analysis is presented in Additional file 1: Data Set S1.

A total of 9 samples from three human donors, recipients and $\mathrm{RpT}$ were collected and transplanted into gnotobiotic mice each as three replicates (27 mouse samples). A total of 36 samples were sequenced with an average sequence depth of 95,464 reads per sample (range 58,972-172877 reads per sample). After quality filtering the sample depth across all samples was normalized to 49,243 reads per sample and used for analysis. A total of 469 operational taxonomic unit (OTU) were identified.

\section{Comparison of donor, recipient and RpT microbe communities}

Comparison of the microbe communities from recipient and donor samples using principle coordinate analysis (PCoA) generated from weighted UniFrac metrics revealed each formed separate clusters (Fig. 1). The microbe communities from the post FMT patients (RpT) generally clustered in between the donor or recipient samples on the PCoA plot. The donor samples showed a high abundance of commensal microbes from the families Bacteriodaceae (mean $25 \%$ ) and Lachnospiraceae (mean 30 \%). The Bacteriodaceae and Lachnospiraceae were almost absent in recipient samples $(<0.01 \%$ of the abundance of the donors). The recipient samples contained Enterobacteriaceae, Lactobacillaceae, Enterococcaceae in relative abundance of $43,11,8 \%$ respectively (these microbes were less that $0.01 \%$ in the donors) (Fig. 2. Additional file 3: Data Set S2). Following FMT, the RpT samples from all three patients at 2-4 weeks showed an increase in the proportional abundance of both Bacteriodaceae and Lachnospiraceae to approximately $7 \%$. In addition, the RpT samples showed a decrease in the abundance of families Enterobacteriaceae, Lactobacillaceae, Enterococcaceae. The Shannon's diversity metric of the recipients (mean $2.63 \pm 0.95$ ) was also found to be significantly lower than the donor population (mean $5.18 \pm 0.62)(p<0.05)$. The RpT samples show increased 


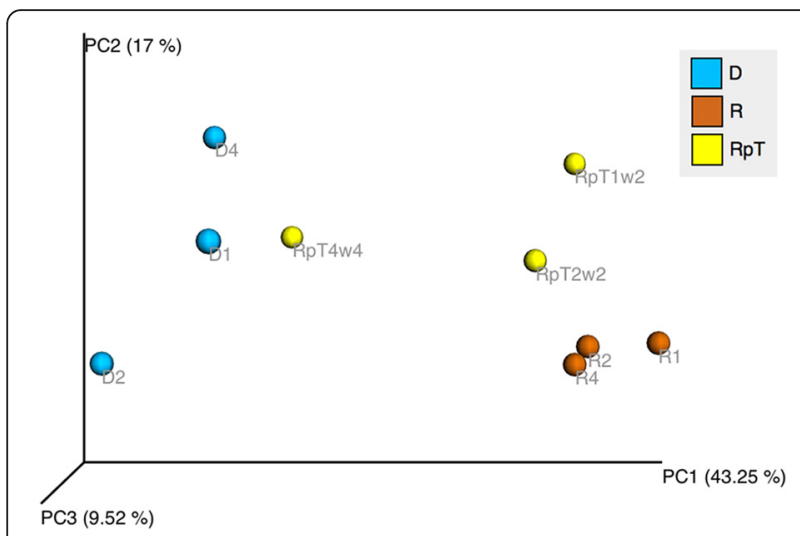

Fig. 1 Comparison of the microbial diversity of human donors and recipients pre and post FMT. Principal Coordinate analysis is used to generate 3D PCoA plot (using weighted UniFrac distance metrics) for the fecal samples from the donor (D) and recipient (R) and post FMT (RpT)

diversity (mean $3.42 \pm 0.97$ ) when compared with the recipients (Additional file 4: Data Set S3). Collectively, our analysis demonstrates that the microbe compositions of the donor and recipient pre FMT are clearly different. Most importantly, the microbe composition 2-4 weeks after FMT in the three recipients differs from both the donor and pre FMT microbiota.

\section{Transplant of donor and recipient pre and post FMT microbiota into gnotobiotic mice}

It has been previously shown that gnotobiotic mice accept and recapitulate the major elements of the human microbiota after transplant $[14,15]$. We wanted to use this feature of gnotobiotic mice to examine the colonization potential of the microbiota from the recipients prior to and after transplant. Following transplant,

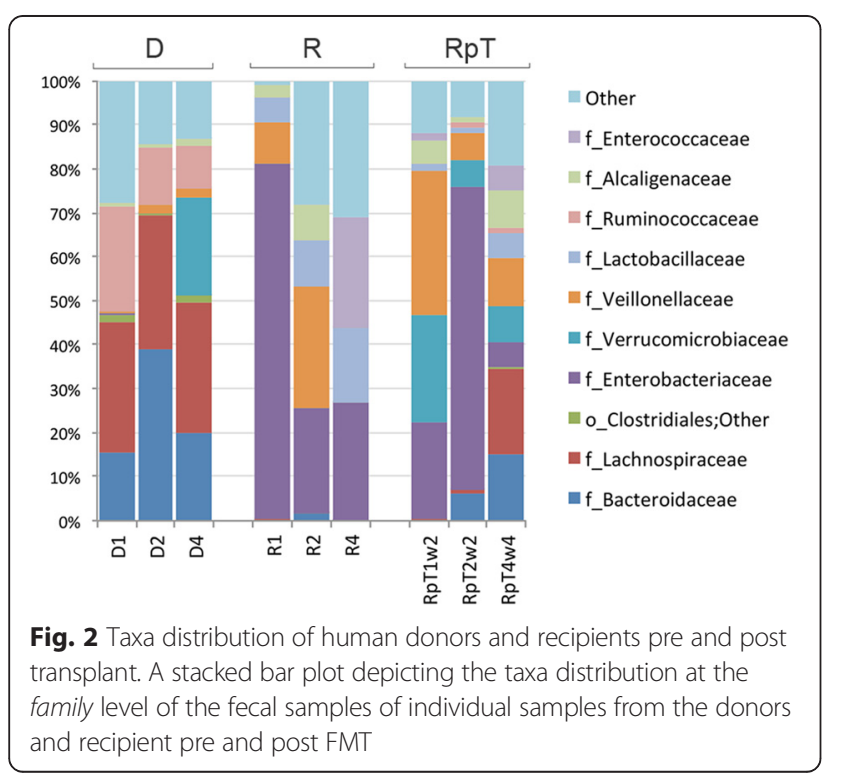

the mice were housed for 4 weeks for equilibration and the microbiome from fecal samples from each mouse was then analyzed.

Similar to what we observed from the analysis of the microbiome composition of the donor and recipient pre FMT, the beta diversity comparison using PCoA plots of the weighted UniFrac analysis showed separate clustering of mouse transplanted donor (MD) from the mouse transplanted recipient (MR) (Fig. 3a). The microbiota of MD samples was found to be statistically different $(p<=0.001)$ than the MR samples. The pattern was consistent with all three cases (Fig. 3b, c and d). The transplantation of the fecal samples from RpT from all three of the FMT patients into gnotobiotic mice (MRpT) resulted in a microbe composition in the mice that clustered with the donor (MD): no statistical difference between MD and MRpT samples was found $(p>0.05)$.

The taxa analysis substantiated the microbe clustering seen on the PCoA plots. In particular, the composition of the MRpT from each of the samples from the mice was similar to the composition of the MD with respect to the abundance of the predominant families of commensal microbiota Bacteroidaceae and Lachnospiraceae (Fig. 4). In contrast, transplantation of the recipient samples into gnotobiotic mice resulted in a microbe composition with an abnormally higher proportion of unclassified Clostridiales (referred to as Clostridiales; Other which includes all Clostridiales which cannot be classified at family level, Additional file 3: Data Set 2) at an average abundance of $55 \%$. This relative abundance is approximately 7000 times increased from the relative abundance in the recipient samples (i.e. pre-transplant into gnotobiotic mice). Thus, the microbes within the unclassified Clostridiales in the recipients have the capacity to colonize and amplify following transplantation into gnotobiotic mice. Further examination of the sequences from the unclassified Clostridiales revealed two major OTUs. One OTU (denovo6164) shows $100 \%$ similarity to $C$. difficile (accession number gi:822490352) while the second OTU (denovo14687) shows $100 \%$ similarity to Bacterium NLAE (accession number gi:379364487). Consistent with the increased abundance of the unclassified Clostridiales in the MR samples, there was a lower Shannon's diversity (2.8) as compared to MD (4.6). The Shannon metric of diversity of the MRpT samples was higher than that of the MR samples and similar to that of the MD (Fig. 5).

To further highlight the commensal microbe expansion in the gnotobiotics, we next compared the relative abundance of the Bacteriodaceae and Lachnospiraceae in the human and mouse transplants. The microbes of the human donor samples demonstrated the capacity of the Bacteriodaceae and Lachnospiraceae to colonize gnotobiotic mice following transplantation (Fig. 6). Even though the RpT samples had lower abundance of Bacteriodaceae 


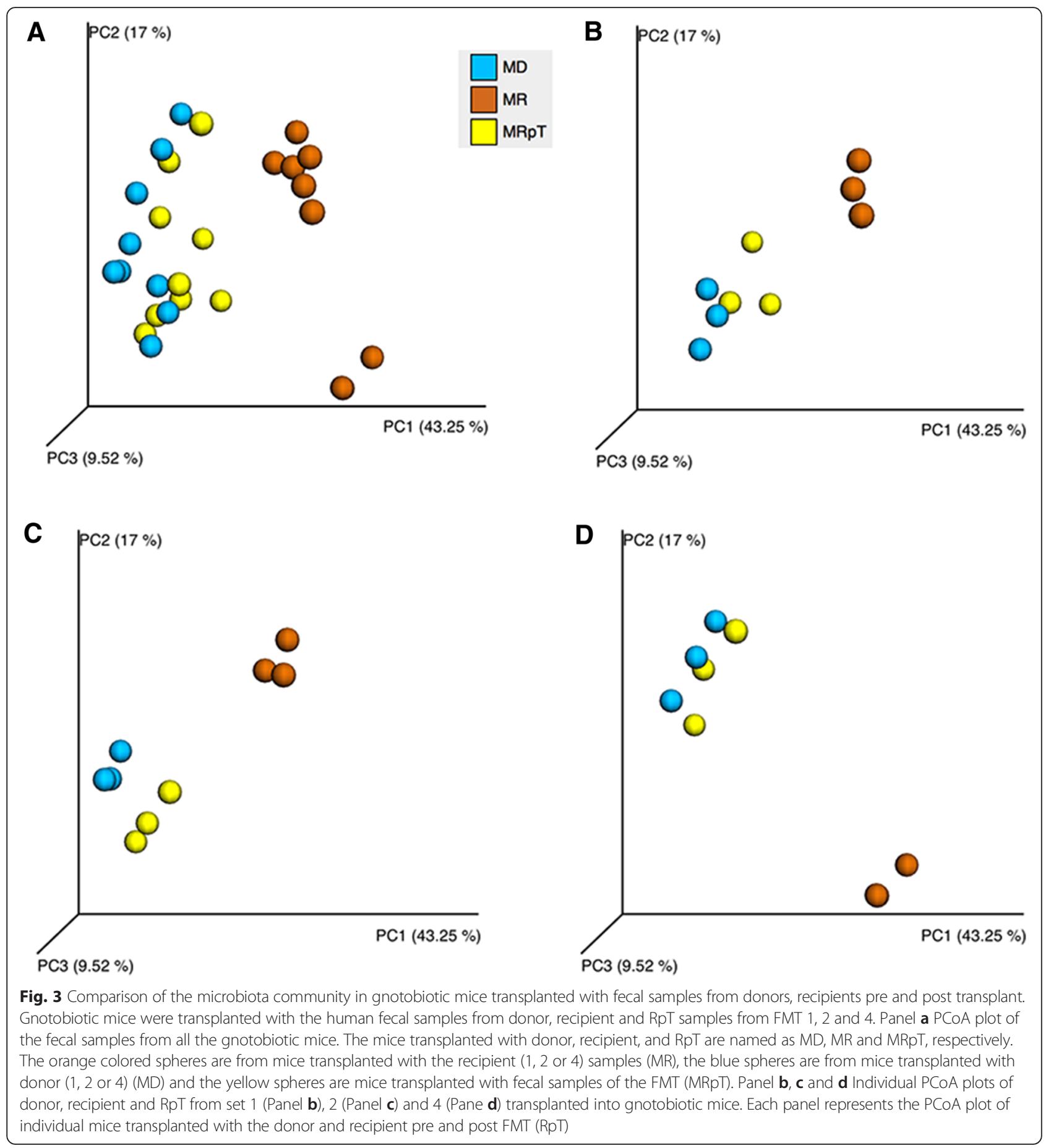

and Lachnospiraceae, the abundance of both increased following transplant into gnotobiotic mice to a similar abundance as gnotobiotic mice transplanted with the donor samples, highlighting the capacity of both Bacteriodaceae and Lachnospiraceae to effectively colonize and even expand following transplantation (Additional file 3: Data Set 2). In parallel, we found the relative abundance of microbes of the unclassified Clostridiales also increased in the MRpT from the human RpT to an average abundance of $10 \%$ from $0.2 \%$ (Data Set 2). However, this increase was only 50 times greater that that found in the human RpT samples as compared to the 7000 times greater for the transplant of the recipient samples in gnotobiotic mice (i.e. human $\mathrm{R}$ to MR). 


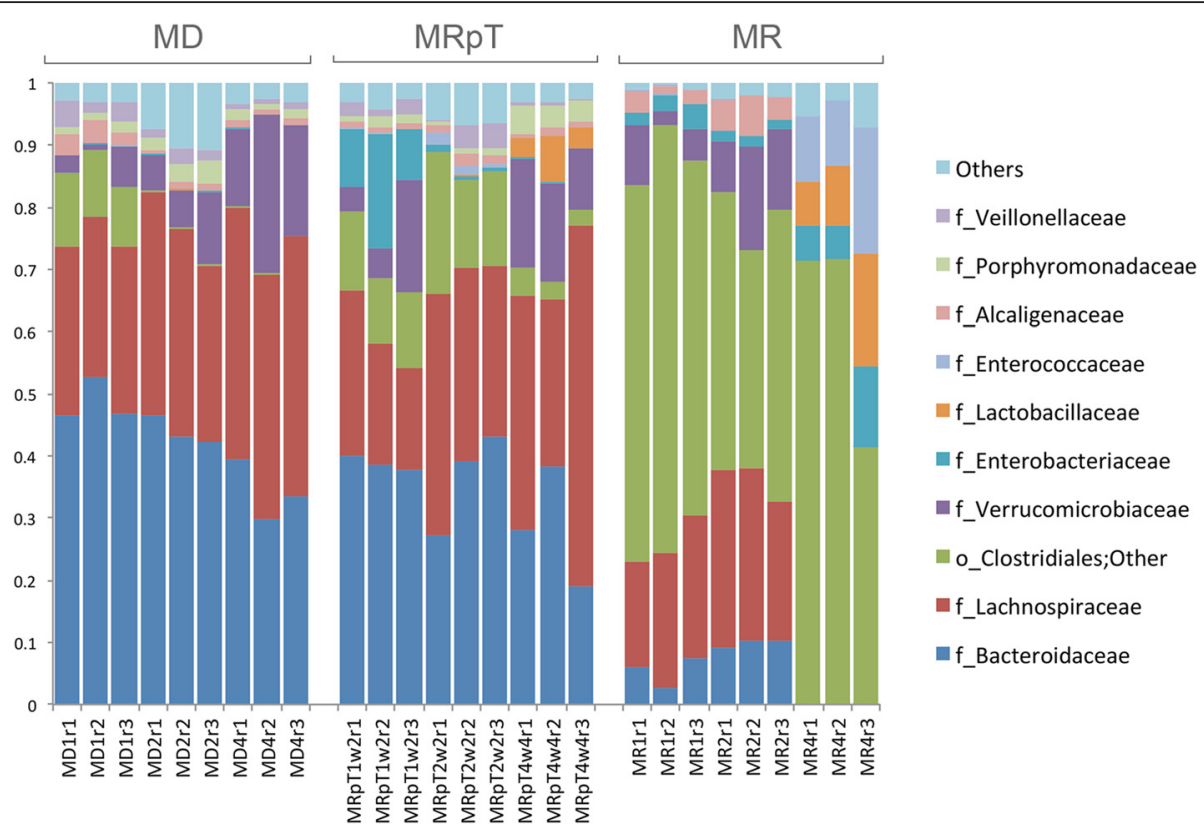

Fig. 4 Taxa distribution of the microbial composition of mouse transplanted with donor, recipient pre and post FMT. A stacked bar plot depicting the taxa distribution at the family level of the fecal samples of individual gnotobiotic mice transplanted with samples from the donors and recipient pre and post FMT

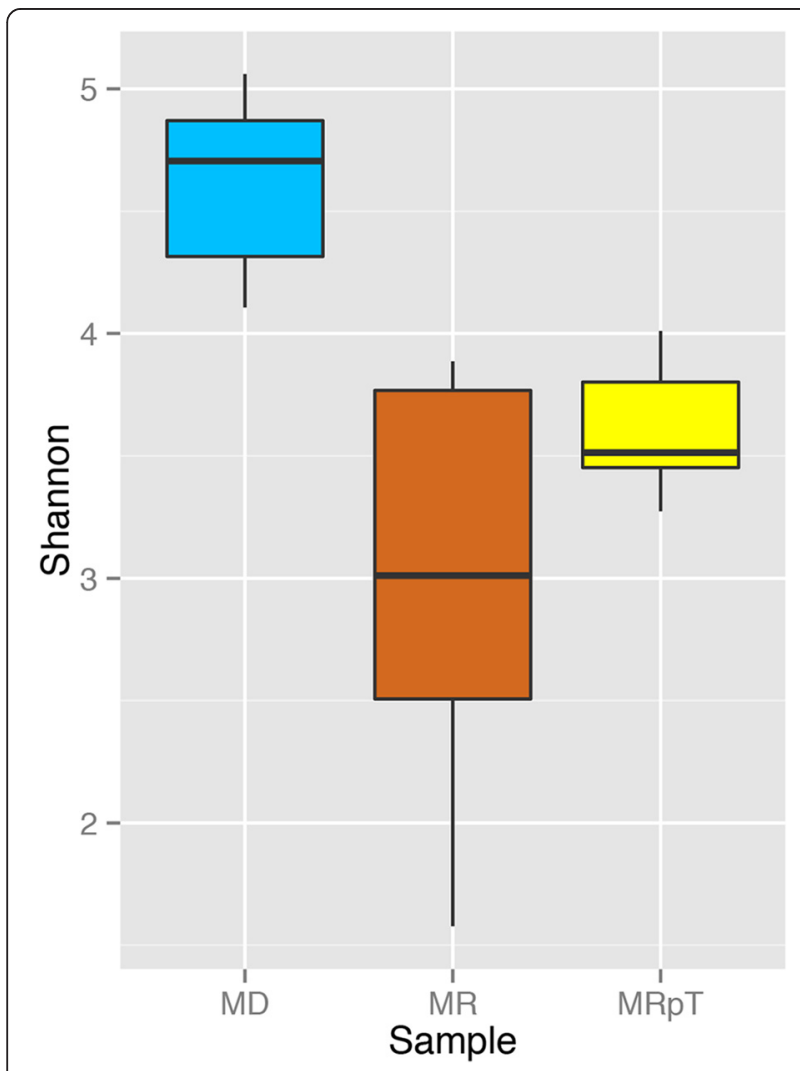

Fig. 5 Shannon diversity for gnotobiotic mice transplanted with donor, recipient pre and post FMT. Alpha diversity (Shannon's diversity) for gnotobiotic mice transplanted with the donor (blue), recipients pre (orange) and post FMT (yellow) microbiota presented as a box plot

\section{Discussion}

Previous studies have analyzed the microbiome of donor and FMT recipient pre and post-transplant [16-19]. Similar to the results we found with our three patients, these studies found that before FMT, the microbial composition of the recipient was of low diversity and different from the donor [16, 18-20]. Similar to our observation, Shahinas et al. studied 2-week post-FMT samples and noted $50 \%$ samples show noticeable early reconstitution [17]. It is not surprising in our study then, that early after FMT the microbe composition of the recipients was different from both the donor and the recipient.

One of the early events for a successful transplant is the ability of the transplanted microbiota to access the gastrointestinal niches [21]. Once the donor microbes reach the niches, they must also compete for nutrients and space to expand their numbers. To gain insights into the reconstitution of the microbial community in the FMT patients, we took advantage of the capacity of gnotobiotic animals since these mice are devoid of microbes and all of the niches in the gastrointestinal tract would be open for colonization [22-24].

We first analyzed the microbe communities in mice transplanted with the donor or recipient fecal samples. The microbial composition of the mice transplanted with the donor did not cluster with that of mice transplanted with the recipients, consistent to what we saw from the analysis of the donor and recipient obtained from humans. Furthermore, we did not find a difference in the relative 


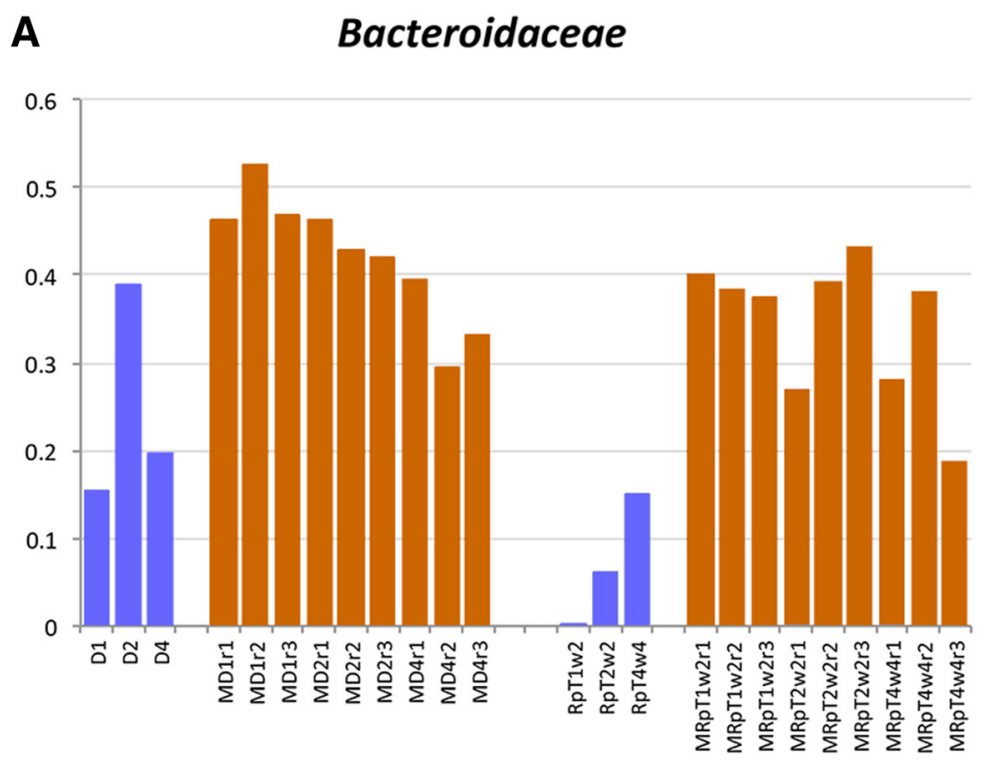

B Lachnospiraceae

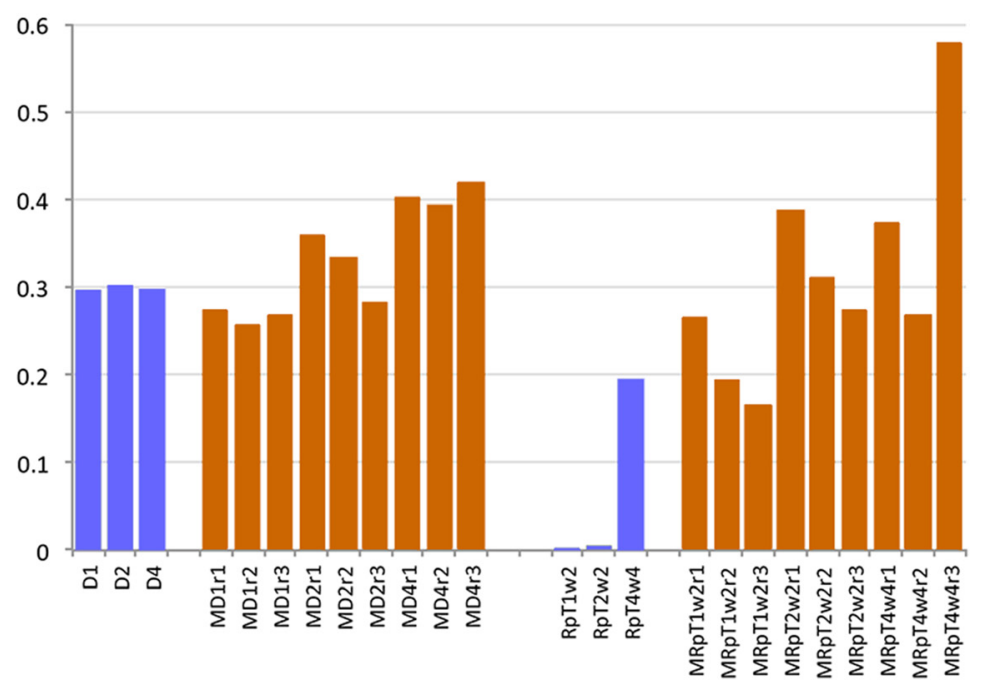

Fig. 6 Comparison of relative abundance of selected taxa (family level). The human samples are colored in blue and the gntobiotic mice samples are colored orange. Panel a The relative abundance of Bacteroides in the human donors and post FMT and their corresponding transplants in gnotobiotic mice. Panel $\mathbf{b}$ The relative abundance of Lachnospiraceae in the human donors and post FMT and their corresponding transplant in gnotobiotic mice

abundance of Bacteroidaceae and Lachnospiraceae in the human and compared to the gnotobioitic. As evidenced from the analysis of the recipient's microbiota, the microbe composition was very different from that of the donor with increased relative abundance of families Enterobacteriaceae, Lactobacillaceae, Enterococcaceae, consistent with a dysbiotic microbe composition $[4,25]$. Transplantation of the recipient fecal samples into gnotobiotic mice though revealed a different composition from that of the recipient with an increase in the relative abundance of the unclassified Clostridiales. Thus, even though the unclassified Clostridiales microbes represented a minor percentage of the recipient microbes, they had the capacity to amplify and occupy the vacant niches in the gntobiotic mice. The reason why these microbes were not major constituents of the recipient microbe population is unknown. One possibility is that the unclassified Clostridiales might have a selective growth advantage in the mouse gut over the other microbes in the recipient's sample (i.e. families Enterobacteriaceae, Lactobacillaceae, Enterococcaceae). Additional experiments will be required to address this possibility.

We next characterized the colonization potential of the recipient's fecal sample at early times post FMT. Even though the microbe composition 2-4 weeks after 
FMT in the three recipients differs from both the donor and pre FMT microbiota, the transplantation of the RpT fecal samples into gnotobiotic animals resulted in a microbe composition that clustered with the donor. We found the proportion of the commensal microbes of the families Bacteroidaceae and Lachnospiraceae were increased following transplantation into the gnotobiotic mice to levels similar to that of the mice transplanted with the donor samples. This result suggests that the commensal microbes, represented by families Bacteroidaceae and Lachnospiraceae, in the RpT transplanted into the gnotobiotic mice with the open niches probably out compete the non-commensal microbes present in the sample after FMT for niche space in the gnotobiotic mice. Support for this conclusion comes from previous studies that have shown commensal gut microbes such as Bacteroides, have evolved species-specific physical interactions with the host that mediate stable and resilient gut colonization [25-27].

One of the important functions of the commensal gut microbes is to provide an environment to protect against over growth of pathogenic microbes [4, 25, 28, 29]. Indeed, the use of multiple antibiotic treatments to treat $C$. difficile is thought to stress the regenerative properties of the gut microbiota that can promote conditions conducive for over growth of pathogens leading to the dysbiotic microbe composition. It was clear though from the analysis of the RpT transplants in gnotobiotic mice that the microbes of the families Bacteroidaceae and Lachnospiraceae had the capacity to reconstruct a microbe community that had the capacity to inhibit the expansion of the unclassified Clostridiales still present in the fecal samples. In the MRpT samples the presence of the microbes of the families $\mathrm{Bac}$ teroidaceae and Lachnospiraceae (abundance $7 \%$ of that found in the donor) resulted in 50 fold expansion of unclassified Clostridiales as opposed to the 7000 fold expansion on the recipient sample that had reduced amounts of the families Bacteroidaceae and Lachnospiraceae (less that $0.01 \%$ of the donor). It is not clear whether the reduced expansion of the unclassified Clostridiales is due solely to the Bacteroidaceae and Lachnospiraceae out competing for niche space since from the studies of the recipient transplants in gnotobiotic mice the unclassified Clostridiales have the capacity to colonize the gnotobiotic mice. Another possibility is that the mouse diet, which is known to impact the gut microbiome composition, might have favored the further expansion of the Bacteroidaceae and Lachnospiraceae over the unclassified Clostridales. Additional studies will be needed to understand the community dynamics that controls the continued reconstruction of the microbe community at these later times following FMT $[4,30]$.

\section{Conclusion}

Numerous studies have reported on the remarkable success of FMT for treatment to resolve dysbiotic microbiomes that are a result of extensive antibiotics to treat $C$. difficile infections $[4,8-11,16,31,32]$. Our results using transplantation of gnotobiotic mice as a model demonstrates the increased colonization potential of commensal microbes (families Bacteroidaceae and Lachnospiraceae) compared to microbes found in the dysbiotic recipient microbiota. The increased colonization potential of these commensal microbes for the gastrointestinal tract provides a framework to understand the success of FMT to reconstitute the gut microbe community of patients with recurrent $C$. difficile infections.

\section{Methods}

\section{Sample collection and processing}

Fecal samples were collected from donors and recipients prior to and post FMT. The samples were processed and archived using $10 \%$ glycerol as a cryopreservative as previously described [33, 34]. Consent form was obtained for FMT and microbiome analysis as part of an ongoing University of Alabama at Birmingham Institutional Review Board (UAB IRB) approved study at the University of Alabama at Birmingham.

\section{Isolation of microbial DNA and creation of $16 \mathrm{~S}$ V4 amplicon library}

Microbial genomic DNA was isolated using the Fecal DNA isolation kit from Zymo Research following the manufacturer's instructions. Once the sample DNA was prepared, PCR was used with unique bar coded primers to amplify the variable region 4 (V4) region of the $16 \mathrm{~S}$ rDNA gene to create an amplicon library from individual samples [33, 35] (Additional file 2: Text S1).

\section{Illumina MiSeq DNA sequencing and bioinformatics}

The PCR product was $\sim 255$ bases from the V4 segment of the 16S rDNA gene, and we sequenced 251 bases single end reads using Illumina MiSeq [33, 35]. FASTQ conversion of the raw data files was performed following demultiplexing using MiSeq reporter. Quality assessment of the FASTQ files was performed using FASTQC [36] and then quality filtering was done using the FASTX toolkit [37]. Due to low quality of single base toward the 3' ends of the read, the last base were trimmed for all reads, making the read length as 250 bases. Any read with an average base quality $\mathrm{Q}$ score of $<20$ and the reads with unknown bases ("N") were discarded. The remainder of the steps was performed with the Quantitative Insight into Microbial Ecology (QIIME) suite, version 1.8 as described below $[35,38,39]$. Chimeric sequences were filtered using the "identify_chimeric_seqs.py" module of USEARCH [40]. Sequences were grouped into operational taxonomic units (OTUs) using the clustering program UCLUST at a similarity threshold of $97 \%$ [40]. The Ribosomal Database Program (RDP) classifier trained using the Greengenes 
(v13.8) 16S rRNA database [41] was used to make taxonomic assignments for all OTUs at confidence threshold of $80 \%$ (0.8) [42]. The resulting OTU table included all OTUs, their taxonomic identification, and abundance information. OTUs whose average abundance was less than $0.005 \%$ were filtered out [43]. OTUs were then grouped together to summarize taxon abundance at different hierarchical levels of classification (e.g. phylum, class, order, family, genus, and species). These taxonomy tables were also used to generate stacked column bar charts of taxon abundance using Microsoft Excel software (Microsoft, Seattle, WA). Multiple sequence alignment of OTUs was performed with PyNAST [44]. Alpha diversity (within sample diversity) was calculated using Shannon's metrics as implemented in QIIME (Additional file 4: Data Set S3) [39]. Beta diversity (between sample diversity) among different samples was measured using weighted UniFrac metrics (distance matrices in Additional file 4: Data Set S3) [45]. Principal coordinates analysis (PCoA) was performed by QIIME to visualize the dissimilarity matrix (beta-diversity) between all the samples. 3D PCoA plots were generated using EMPEROR [46].

\section{Statistical analysis}

Samples were grouped into donor, recipient and transplants for both mouse and human. Differences of taxa abundance and alpha diversity between two groups are measured using unpaired T-test (assuming unequal variance) and considered significant at $p<0.05$. Differences in microbiota between groups are measured using PERMANOVA (weighted UniFrac distance).

\section{Transplantation of archived fecal samples into gnotobiotic mice}

Germ-free C57BL/6 mice (3 mice/group) were colonized with archived fecal samples. The sample was first defrosted on ice and a total of 200ul was delivered via the oral and intra-colonic routes to each mouse. Animals with the same fecal samples were housed in the same isolator. Food was autoclaved mouse chow and supplied ad libitum. In preliminary experiments, we determined that 2 weeks post transplant was sufficient to allow re-constitution of an intact microbiome. For the current study, fecal samples were taken at 4 weeks. The pellets were processed for DNA and $16 \mathrm{~S}$ rDNA microbiome analysis is performed. All mice were bred and maintained and all animal experimentation was approved in accordance with guidelines and approval of the University of Alabama at Birmingham Institutional Animal Care and Use Committee.

\section{Additional files}

Additional file 1: Characteristics of donors and patients. (XLSX $12 \mathrm{~kb})$
Additional file 2: Preparation of the donor sample and administration. (DOCX $100 \mathrm{~kb}$ )

Additional file 3: Relative abundance of microbes at family level taxonomy. (XLSX $69 \mathrm{~kb}$ )

Additional file 4: Alpha and beta diversity metrics. (XLSX $25 \mathrm{~kb}$ )

\section{Abbreviations}

FMT: fecal microbiota transplant; D: donor; R: recipient; RpT: recipient post transplant; w: week; M: mouse; OTU: operational taxonomic unit; PCoA: principle coordinate analysis; IRB: institutional review board; $\mathrm{V} 4$ : variable region 4 of the rDNA gene; QIIME: quantitative insight into microbial ecology; RDP: ribosomal database program

\section{Competing interests}

The authors declare that they have no competing interests.

\section{Authors' contributions}

RK, TP, DKC and EJL did the bioinformatics and statistics, CLM and CTW transplanted the gnotobiotic mice and participated in the experimental design, PE, KTG and MRC carried out the $16 \mathrm{~S}$ rDNA amplification and DNA sequencing, JAG, PD, WHB Jr. and JMR did the FMT and coordinated collection of samples and RK, CLM, JMR and CDM conceived of the study, and participated in its design and coordination and helped to draft the manuscript. All authors read and approved the final manuscript.

\section{Acknowledgements}

The following are acknowledged for their support of the Microbiome Resource at the University of Alabama at Birmingham: School of Medicine, Comprehensive Cancer Center (P30AR050948), Center for AIDS Research (5P30AI027767), Center for Clinical Translational Science (UL1TR000165) and Heflin Center. We thank Adrienne Ellis for preparation of the manuscript.

\section{Author details}

${ }^{1}$ Center for Clinical and Translational Sciences, University of Alabama at Birmingham, Birmingham, AL 35294, USA. Department of Pathology, University of Alabama at Birmingham, Birmingham, AL 35294, USA.

${ }^{3}$ Department of Cell, Developmental and Integrative Biology, University of Alabama at Birmingham, 1918 University Blvd. MCLM 680, Birmingham, AL 35294, USA. ${ }^{4}$ Department of Genetics and Heflin Center for Genomic Science, University of Alabama at Birmingham, Birmingham, AL 35294, USA. ${ }^{5}$ Department of Microbiology, University of Alabama at Birmingham, Birmingham, AL 35294, USA. ${ }^{6}$ Division of Infectious Disease, Department of Medicine, University of Alabama at Birmingham, Birmingham, AL 35294, USA.

Received: 24 August 2015 Accepted: 29 December 2015

Published online: 13 January 2016

\section{References}

1. He M, Miyajima F, Roberts P, Ellison L, Pickard DJ, Martin MJ, et al. Emergence and global spread of epidemic healthcare-associated Clostridium difficile. Nat Genet. 2013:45:109-13.

2. Jagai J, Naumova E. Clostridium difficile-associated disease in the elderly, United States. Emerg Infect Dis. 2009:15:343-4.

3. Kyne L, Hamel MB, Polavaram R, Kelly CP. Health care costs and mortality associated with nosocomial diarrhea due to Clostridium difficile. Clin Infect Dis. 2002;34:346-53.

4. Seekatz AM, Young VB. Clostridium difficile and the microbiota. J Clin Invest. 2014;124:4182-9.

5. Louie TJ, Miller MA, Mullane KM, Weiss K, Lentnek A, Golan Y, et al. Fidaxomicin versus vancomycin for Clostridium difficile infection. N Engl J Med. 2011:364:422-31.

6. Musher DM, Aslam S, Logan N, Nallacheru S, Bhaila I, Borchert F, et al. Relatively poor outcome after treatment of Clostridium difficile colitis with metronidazole. Clin Infect Dis. 2005:40:1586-90.

7. Pepin J, Routhier S, Gagnon S, Brazeau I. Management and outcomes of a first recurrence of Clostridium difficile-associated disease in Quebec, Canada. Clin Infect Dis. 2006;42:758-64.

8. Kelly CP. Fecal microbiota transplantation-an old therapy comes of age. N Engl J Med. 2013;368:474-5. 
9. Landy J, Al-Hassi HO, McLaughlin SD, Walker AW, Ciclitira PJ, Nicholls RJ, et al. Review article: faecal transplantation therapy for gastrointestinal disease. Aliment Pharmacol Ther. 2011;34:409-15.

10. Rohlke F, Stollman N. Fecal microbiota transplantation in relapsing Clostridium difficile infection. Ther Adv Gastroenterol. 2012;5:403-20.

11. Rohlke F, Surawicz CM, Stollman N. Fecal flora reconstitution for recurrent Clostridium difficile infection: results and methodology. J Clin Gastroenterol. 2010;44:567-70

12. Rupnik M, Wilcox MH, Gerding DN. Clostridium difficile infection: new developments in epidemiology and pathogenesis. Nat Rev Microbiol. 2009; 7:526-36.

13. Dunne C. Adaptation of bacteria to the intestinal niche: probiotics and gut disorder. Inflamm Bowel Dis. 2001;7:136-45.

14. Faith JJ, McNulty NP, Rey FE, Gordon Jl. Predicting a human gut microbiota's response to diet in gnotobiotic mice. Science. 2011;333:101-4.

15. Goodman AL, Kallstrom G, Faith JJ, Reyes A, Moore A, Dantas G, et al. Extensive personal human gut microbiota culture collections characterized and manipulated in gnotobiotic mice. Proc Natl Acad Sci U S A. 2011;108:6252-7.

16. Petrof EO, Gloor GB, Vanner SJ, Weese SJ, Carter D, Daigneault MC, et al. Stool substitute transplant therapy for the eradication of Clostridium difficile infection: 'RePOOPulating' the gut. Microbiome. 2013:1:3.

17. Shahinas D, Silverman M, Sittler T, Chiu C, Kim P, Allen-Vercoe E, et al. Toward an understanding of changes in diversity associated with fecal microbiome transplantation based on 165 rRNA gene deep sequencing. MBio. 2012;3(5): e00338-12:1-7.

18. Shankar V, Hamilton MJ, Khoruts A, Kilburn A, Unno T, Paliy O, et al. Species and genus level resolution analysis of gut microbiota in Clostridium difficile patients following fecal microbiota transplantation. Microbiome. 2014;2:13.

19. Song Y, Garg S, Girotra M, Maddox C, von Rosenvinge EC, Dutta A, et al. Microbiota dynamics in patients treated with fecal microbiota transplantation for recurrent Clostridium difficile infection. PLoS One. 2013;8:e81330.

20. Charrier C, Duncan GJ, Reid MD, Rucklidge GJ, Henderson D, Young P, et al. A novel class of CoA-transferase involved in short-chain fatty acid metabolism in butyrate-producing human colonic bacteria. Microbiology. 2006;152:179-85.

21. Nava GM, Friedrichsen HJ, Stappenbeck TS. Spatial organization of intestinal microbiota in the mouse ascending colon. ISME J. 2011;5:627-38.

22. Freter $R$, Abrams GD. Function of various intestinal bacteria in converting germfree mice to the normal state. Infect Immun. 1972;6:119-26.

23. Fukushima K, Sasaki I, Ogawa H, Naito H, Funayama Y, Matsuno S. Colonization of microflora in mice: mucosal defense against luminal bacteria. J Gastroenterol. 1999:34:54-60.

24. Gillilland 3rd MG, Erb-Downward JR, Bassis CM, Shen MC, Toews GB, Young VB, et al. Ecological succession of bacterial communities during conventionalization of germ-free mice. Appl Environ Microbiol. 2012;78:2359-66.

25. Backhed F, Ley RE, Sonnenburg JL, Peterson DA, Gordon Jl. Host-bacterial mutualism in the human intestine. Science. 2005:307:1915-20.

26. Lee SM, Donaldson GP, Mikulski Z, Boyajian S, Ley K, Mazmanian SK. Bacterial colonization factors control specificity and stability of the gut microbiota. Nature. 2013:501:426-9.

27. Pedron T, Mulet C, Dauga C, Frangeul L, Chervaux C, Grompone G, et al. A crypt-specific core microbiota resides in the mouse colon. MBio. 2012;3(3): e00116-12: 1-7.

28. Kamada N, Chen GY, Inohara N, Nunez G. Control of pathogens and pathobionts by the gut microbiota. Nat Immunol. 2013:14:685-90.

29. Vollaard EJ, Clasener HA. Colonization resistance. Antimicrob Agents Chemother. 1994;38:409-14.

30. Faith JJ, Guruge JL, Charbonneau M, Subramanian S, Seedorf H, Goodman $\mathrm{AL}$, et al. The long-term stability of the human gut microbiota. Science. 2013:341:1237439.

31. Silverman MS, Davis I, Pillai DR. Success of self-administered home fecal transplantation for chronic Clostridium difficile infection. Clin Gastroentero Hepatol. 2010;8:471-3.

32. van Nood E, Vrieze A, Nieuwdorp M, Fuentes S, Zoetendal EG, de Vos WM et al. Duodenal infusion of donor feces for recurrent Clostridium difficile. N Engl J Med. 2013;368:407-15.

33. Kumar R, Eipers P, Little RB, Crowley M, Crossman DK, Lefkowitz EJ, et al Getting started with microbiome analysis: sample acquisition to bioinformatics. Curr Protoc Hum Genet. 2014;82:18 18 11-29.

34. Satokari R, Mattila E, Kainulainen V, Arkkila PET. Simple faecal preparation and efficacy of frozen inoculum in faecal microbiota transplantation for recurrent Clostridium difficile infection - an observational cohort study. Aliment Parmacol Ther. 2015:41:46-53.

35. Kozich JJ, Westcott SL, Baxter NT, Highlander SK, Schloss PD. Development of a dual-index sequencing strategy and curation pipeline for analyzing amplicon sequence data on the MiSeq Illumina sequencing platform. Appl Environ Microbiol. 2013;79:5112-20.

36. FASTQC. http://www.bioinformatics.bbsrc.ac.uk/projects/fastqc. Accessed 1 Sep 2014.

37. FASTX-Toolkit. http://hannonlab.cshl.edu/fastx_toolkit. Accessed 1 Sep 2014

38. Lozupone CA, Hamady M, Kelley ST, Knight R. Quantitative and qualitative beta diversity measures lead to different insights into factors that structure microbial communities. Appl Environ Microbiol. 2007;73:1576-85.

39. Navas-Molina JA, Peralta-Sanchez JM, Gonzalez A, McMurdie PJ, VazquezBaeza Y, Xu Z, et al. Advancing our understanding of the human microbiome using QIIME. Methods Enzymol. 2013:531:371-444.

40. Edgar RC. Search and clustering orders of magnitude faster than BLAST. Bioinformatics (Oxford, England). 2010;26:2460-1.

41. McDonald D, Price MN, Goodrich J, Nawrocki EP, DeSantis TZ, Probst A, et al. An improved Greengenes taxonomy with explicit ranks for ecological and evolutionary analyses of bacteria and archaea. ISME J. 2012;6:610-8.

42. Wang Q, Garrity GM, Tiedje JM, Cole JR. Naive Bayesian classifier for rapid assignment of rRNA sequences into the new bacterial taxonomy. Appl Environ Microbiol. 2007;73:5261-7.

43. Bokulich N, Subramanian S, Faith JJ, Gevers D, Gordon JI, Knight R, et al. Quality-filtering vastly improves diversity estimates from Illumina amplicon sequencing. Nat Methods. 2013:10:57-9.

44. Caporaso JG, Bittinger K, Bushman FD, DeSantis TZ, Andersen GL, Knight R. PyNAST: a flexible tool for aligning sequences to a template alignment. Bioinformatics (Oxford, England). 2010;26:266-7.

45. Lozupone C, Hamady M, Knight R. UniFrac-an online tool for comparing microbial community diversity in a phylogenetic context. BMC Bioinformatics. 2006;7:371.

46. Vazquez-Baeza Y, Pirrung M, Gonzalez A, Knight R. EMPeror: a tool for visualizing high-throughput microbial community data. Gigascience. 2013;2:16.

\section{Submit your next manuscript to BioMed Central and we will help you at every step:}

- We accept pre-submission inquiries

- Our selector tool helps you to find the most relevant journal

- We provide round the clock customer support

- Convenient online submission

- Thorough peer review

- Inclusion in PubMed and all major indexing services

- Maximum visibility for your research

Submit your manuscript at www.biomedcentral.com/submit
) Biomed Central 